\title{
Correlative Microscopic, Spectroscopic, and Computational Analysis of the Nucleation and Growth of Europium (III) Oxalate Nanoparticles
}

\author{
Jennifer A. Soltis ${ }^{1}$, Michele A. Conroy ${ }^{1}$, William C. Isley III $^{1}$, Gabriel B. Hall ${ }^{1}$, Sayandev Chatterjee ${ }^{1}$, \\ Zheming Wang ${ }^{1}$, Shawn M. Kathmann ${ }^{1}$, James J. De Yoreo ${ }^{1}$, Edgar C. Buck ${ }^{1}$, and Gregg J. Lumetta ${ }^{1}$ \\ 1. Pacific Northwest National Lab, Richland, WA 99354, USA
}

The early stages of nanoparticle nucleation are not well understood, and yet increasing our understanding of these processes is fundamental to the production of monodisperse nanoparticles with targeted properties. Many technical challenges exist when trying to study nucleation events due to both the nuclei's small size and rapid formation, and subsequent rapid growth into post-nucleation structures. Additionally, meaningful characterization of nucleation processes must be performed on samples in their in situ solution state. A multi-pronged characterization approach is critical, and experiments are ideally paired with computational modeling to form a more robust picture of these early stages of nanoparticle growth.

The study of europium and other lanthanide oxalate nanoparticles opens many avenues to further work in industrial fields. Lanthanide oxalates may serve as a route through which lanthanide oxide particle shape can be controlled. Lanthanide oxides are used extensively in electronics, optics, and catalysis. The use of a lanthanide oxalate precursor may allow one to circumvent kinetically controlled lanthanide oxide syntheses that produce particles with undesirable size or shape characteristics, thereby expanding the library of shape-controlled lanthanide oxide nanoparticles. Europium (III) was chosen for these investigations because of its convenient spectral properties (e.g., fluorescence behavior) which can provide information complimentary to the electron microscopy studies.

Here we combine scanning (SEM), scanning transmission (STEM), and transmission electron microscopy (TEM), spectroscopy, and computational modeling to elucidate the mechanism of europium (III) oxalate nucleation and growth. Fluid cell SEM and TEM were used to directly image nanoparticle growth in situ and provide a real-time view of the nucleation and growth processes. These results were compared to images of cryogenic (cryo) TEM specimens prepared under similar conditions in order to identify possible differences in growth due to electron beam interactions with the fluid cell samples. Attenuated total reflectance Fourier transform infrared (ATR FTIR) and fluorescence spectroscopies were employed to monitor and identify the onset of Eu-oxalate bond formation and aid in characterizing the amorphous or crystalline nature of structures viewed in the electron micrographs. Finally, computational modeling was used to simulate spectroscopic results from the known crystal structure and provide a reference spectrum for this little-studied material.

Our work shows that europium (III) oxalate synthesis is exquisitely sensitive to reaction conditions such as the concentrations and molar ratio of reactants, $\mathrm{pH}$, and temperature, and varying these parameters leads to differences in particle shape, size, and size distribution and the kinetics of particle growth. Our use of correlative techniques permits an in-depth examination of the structure, morphology, and composition of these particles. Figure 1 shows a scanning electron micrograph of europium (III) oxalate crystals and the corresponding ATR FTIR spectrum, which is in good agreement with simulated and previously published spectra [1]. Europium is evenly distributed throughout the crystals, as seen in the high angle annular dark field (HAADF) STEM image in Figure 2a. Figure 2b shows a high resolution TEM image in which the lattice fringes indicate the presence of several slightly misaligned crystal 
domains, supporting the hypothesis that the overall europium (III) oxalate structure is composed of many crystalline subunits.

Our preliminary results have shown in situ electron microscopy and cryo-TEM, coupled with vibrational and electronic spectral tools, show much promise for understanding how solution conditions influence $\mathrm{Eu}(\mathrm{III})$ oxalate nucleation, and subsequent crystal growth. These insights could lead to new routes to lanthanum oxide materials with tailored properties [2].

\section{References:}

[1] N. Vigier et al, Journal of Alloys and Compounds 444-445 (2007) p. 594-597.

[2] Authors acknowledge support by the Laboratory Directed Research and Development funds of Pacific Northwest National Laboratory (PNNL). PNNL is operated for the U.S. Department of Energy by Battelle Memorial Institute under Contract No. DE-AC06-76RLO 1830.
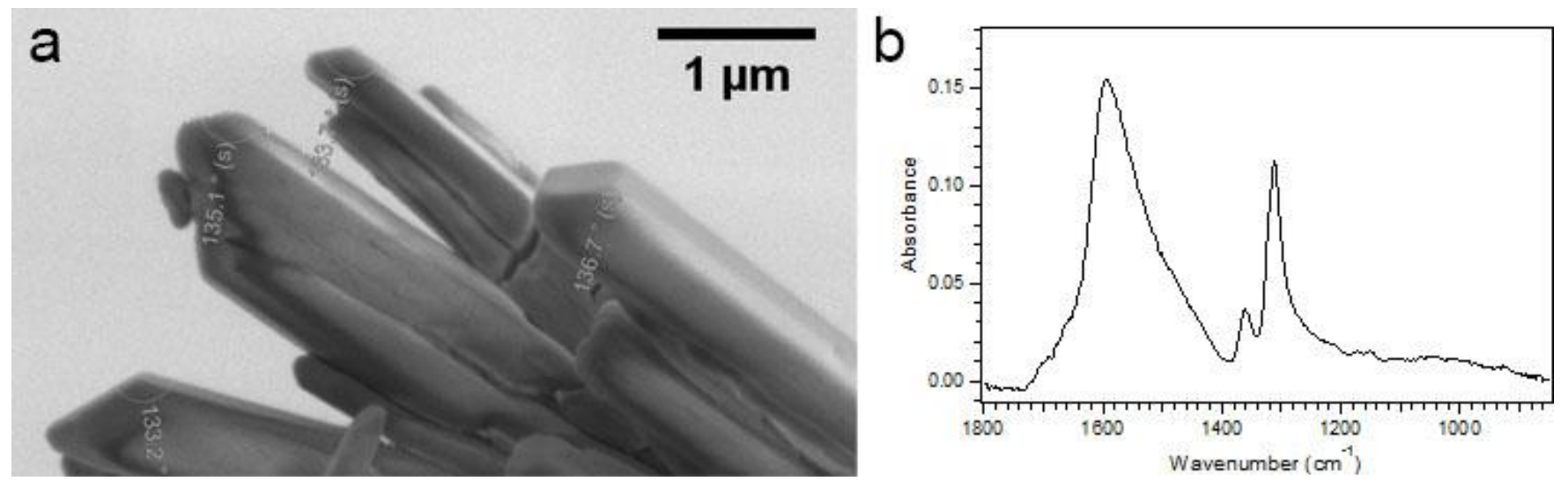

Figure 1. (a) SEM image and (b) ATR FTIR spectrum of europium (III) oxalate crystals.

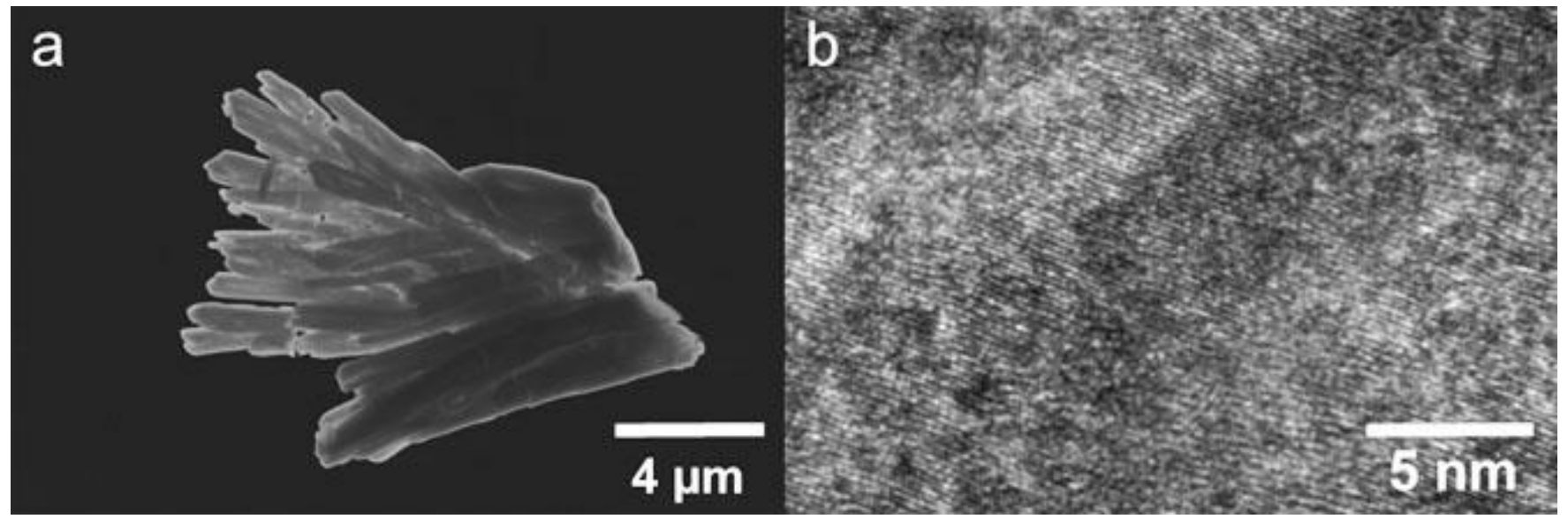

Figure 2. (a) HAADF STEM and (b) high resolution TEM images of europium (III) oxalate crystals. 